\title{
Correction: Design, implementation, and evaluation of a nurse-led intravitreal injection programme for retinal diseases in Singapore
}

\author{
Alvin Wei Jun Teo $($ Tyler Hyungtaek Rim - Chee Wai Wong • Andrew Shih Hsiang Tsai • Nazurah Loh • \\ Thiyagarajan Jayabaskar · Tien Yin Wong · Chui Ming Gemmy Cheung • lan Yew San Yeo
}

Published online: 6 July 2020

(c) The Author(s), under exclusive licence to The Royal College of Ophthalmologists 2020

Correction to: Eye

https://doi.org/10.1038/s41433-020-0920-2

In the original Article, the authors said that there are no nurse led IVT injections provided in Asia in the summary ("Nurse administered intravitreal injection services are slowly gaining acceptance to increase treatment capacity but no such services have been reported in Asia") but in doing so inadvertently left out their fellow eye centre in Singapore, "The Eye Institute, Tan Tock Seng Hospital (TTSH)", who have successfully implemented the practice with their own training protocol in 2017.

The authors have attached the news article that reported on this and would like to apologise for not including this in the review/findings of this practice in our article. The original article has been changed as follows:
The words "outside Singapore" have been added to the last paragraph of the introduction section. That passage now reads:

"In Asia, where the traditional roles of the physician and nurse are well demarcated and patients may prefer treatment by physicians, we are not aware of any NLIVT programme outside Singapore".

The other modified sentence appears in the Summary. It now reads:

"Nurse administered intravitreal injection services are slowly gaining acceptance to increase treatment capacity and apart from few centres like Tan Tock Seng Hospital (TTSH) in Singapore, who have launched this service in 2017, no other such services have been reported in Asia". 